\title{
SIMULAÇÃO NUMÉRICA DO FLUXO RECIRCULATÓRIO EM UM REATOR RH: APLICAÇÃO À DESSULFURAÇÃO*
}

\author{
Thais Couto Silva ${ }^{1}$ \\ Eliana Ferreira Rodrigues ${ }^{2}$ \\ Cláudio Soares ${ }^{3}$ \\ Carlos Antônio da Silva \\ Itavahn Alves da Silva
}

\section{Resumo}

Foram realizadas simulações numéricas, utilizando Computational Fluid Dynamics, para determinar o comportamento fluidodinâmico em condições isotérmicas de metal e gás no interior de um reator $\mathrm{RH}$. São estudados os efeitos da taxa de injeção de gás sobre a distribuição espacial de gás, taxa de circulação e campo de velocidade no sistema. Analisa-se, a partir dos dados de simulação, os resultados de uma operação industrial de dessulfuração por adição de reagentes na câmara de vácuo.

Palavras-chave: Reator $\mathrm{RH}$; Modelagem; Dessulfuração.

\section{NUMERICAL SIMULATION OF RECIRCULATING FLOW IN RH REACTOR: APPLICATION TO DESULFURIZATION}

\section{Abstract}

Computational Fluid Dynamics has been applied in order to describe the flow of gas and metal inside a $\mathrm{RH}$ reactor. The effects of gas injection on gaseous spatial distribution, steel circulation rate and flow field inside the ladle, snorkels and vacuum camera have been assessed. Data from this simulation were used for analyzing the results of a desulfurization trial performed by addition of reagents inside the vacuum camera.

Keywords: $\mathrm{RH}$ reactor; Modeling; Desulfurization.

1 Graduanda, Eng. Metalúrgica, Escola de Minas, Universidade Federal de Ouro Preto (UFOP), Ouro Preto, MG, Brasil.

Engenheira Mecânica, DSc. Professora, Escola de Minas, UFOP, Ouro Preto, MG, Brasil.

3 Engenheiro Metalúrgico, Companhia Siderúrgica do Pecém (CSP), São Gonçalo do Amarante, CE, Brasil.

4 Engenheiro Metalúrgico, Ph.D., Professor, DEMET, Escola de Minas, UFOP, Ouro Preto, MG, Brasil.

5 Engenheiro Metalúrgico, DSc., Professor, DEMET, Escola de Minas, UFOP, Ouro Preto, MG, Brasil. 


\section{1 - INTRODUÇÃO}

O processo $\mathrm{RH}$ (Ruhrstal Heraeus), originalmente desenvolvido para desidrogenação do aço líquido, tem tido larga aplicação como reator metalúrgico de descarburação, desoxidação, controle e homogeneização composicional. Vários pesquisadores [1-3], entre outros ressaltam que a cinética destas reações, são afetadas principalmente pela taxa de circulação do aço entre a câmara de vácuo e a panela. Salientam que a taxa de circulação depende da vazão de argônio, posicionamento e configuração dos bicos de injeção de argônio; número, comprimento, diâmetro interno e formato das pernas; pressão na câmara de vácuo. A literatura disponibiliza correlações para o cálculo da taxa de circulação, tais como:

De acordo com Kuwabara [2]

$$
\mathrm{Q}(\text { ton } / \mathrm{min})=114 \mathrm{G}^{1 / 3} \mathrm{~d}^{4 / 3}\left(\ln \frac{\mathrm{P}_{1}}{\mathrm{P}_{2}}\right)^{1 / 3} \sim 113
$$

$\mathrm{Q}=$ taxa de circulação (ton/min); $\mathrm{d}=$ diâmetro das pernas $(\mathrm{m}) ; \mathrm{G}=$ taxa de injeção de gás $\left(\mathrm{Nm}^{3} / \mathrm{min}\right) ; \mathrm{P}_{1}=$ pressão na câmara de vácuo; $\mathrm{P}_{2}=$ pressão de injeção. Enquanto que, Seshadri e Costa [4] apontam que:

$$
Q=5,89 G^{0,33}
$$

$\mathrm{Q}=$ taxa de circulação do aço (ton/min); $\mathrm{G}$ = a taxa de injeção de gás inerte na perna de subida (NI/min). Já Silva et al. [3] estabelece que:

$$
Q k(g / s)=0,1815 \frac{H_{i m}^{0,607} D^{0,665} \varepsilon^{0,389}}{H^{0,102}}
$$

$\mathrm{H}_{\mathrm{im}}=$ imersão das pernas, $\mathrm{m} ; \mathrm{D}=$ diâmetro das pernas, $\mathrm{cm} ; \varepsilon=$ agitação induzida pelo fluxo de argônio , $\mathrm{W} / \mathrm{kg} ; \mathrm{H}$ = altura de líquido na câmara de vácuo, $\mathrm{cm}$.

A análise da influência do campo de velocidades sobre fenômenos como remoção de inclusões, descarburação, pode também ser encontrada na literatura.

\section{2 - MATERIAIS E MÉTODOS}

As dimensões do reator industrial, utilizadas nesta simulação estão elencadas na Tabela 1. São 12 bicos injetores de gás argônio.

Tabela 1: Dimensões da geometria criada.

\begin{tabular}{cccc}
\hline Panela & Mm & Pernas & $\mathrm{mm}$ \\
\hline $\begin{array}{c}\text { Diâmetro inferior da } \\
\text { panela } \\
\begin{array}{c}\text { Diâmetro superior da } \\
\text { panela }\end{array}\end{array}$ & 3066,6 & Diâmetro Interno & 650,0 \\
$\begin{array}{c}\text { Altura da panela } \\
\text { Altura da panela com } \\
\text { líquido (excluindo } \\
\text { borda livre) }\end{array}$ & 3561,0 & Diâmetro Externo & 1050,0 \\
Câmara de vácuo & 3948,0 & Altura & 1644,0 \\
Diâmetro & 3520,00 & $\begin{array}{c}\text { Profundidade de } \\
\text { imersão }\end{array}$ & 950,0 \\
Altura & Mm & Injetores & $\mathrm{mm}$ \\
\hline & 2950,0 & $\begin{array}{c}\text { Diâmetro do bico } \\
\text { Altura de injeção } \\
\text { (distância em relação } \\
\text { ao fundo da perna) }\end{array}$ & 3,0 \\
\end{tabular}


Para as simulações matemáticas foram empregados os softwares Ansys CFX e SolidWorks. Os modelos de turbulência utilizados foram o SST ou k- $\varepsilon$. Empregou-se uma malha tetraédrica com cerca de 245.000 nós. A técnica de inflation foi empregada nas proximidades das paredes da câmara a vácuo, da panela; pernas de subida e descida. Foi tomado proveito da simetria do $\mathrm{RH}$ para reduzir os custos de computação. A simulação foi conduzida para condição de escoamento turbulento isotérmico aço/argônio, considerando estes como sendo fluidos incompressíveis. As seguintes condições operacionais foram adotadas: $1600^{\circ} \mathrm{C}$, pressão na câmara de vácuo de 50mbar; pressão atmosférica na panela de aço.

As propriedades físicas dos fluidos, aço e argônio estão listadas na Tabela 2.

Tabela 2: Propriedades Físicas dos Fluidos.

\begin{tabular}{lcc}
\hline & Aço Líquido & Argônio \\
\hline Massa específica $\left(\mathrm{kg} \mathrm{m}^{-3}\right)$ & 7000 & 0,568 \\
Temperatura $\left({ }^{\circ} \mathrm{C}\right)$ & 1650 & 25 \\
Viscosidade Dinâmica $(\mathrm{Pa} \mathrm{s})$ & $5,7 \times 10^{-3}$ & $4,848 \times 10^{-5}$ \\
Calor Específico $\left(\mathrm{J} \mathrm{kg}^{-1} \mathrm{k}^{-1}\right)$ & 680 & 15 \\
Condutividade Térmica $\left(\mathrm{W} \mathrm{m}^{-1} \mathrm{k}^{-1}\right)$ & 26 & 5 \\
Tensão Superficial $\left(\mathrm{N} \mathrm{m}^{-1}\right)$ & & 1,54 \\
\hline
\end{tabular}

Para a modelagem matemática adotou-se escoamento multifásico EurelianoEureliano para um modelo não homogêneo. Na região bifásica, a formulação VOF (fração de volume) foi usada de modo a contabilizar as interações entre as interfaces das fases gás/aço líquido. As seguintes equações foram resolvidas (maior detalhamento pode ser encontrado no manual do Ansys-CFX):

Equação da continuidade;

Equação da conservação do volume;

$$
\frac{\partial\left(r_{\alpha} \rho_{\alpha}\right)}{\partial t}+\nabla .\left(r_{\alpha} \rho_{\alpha} U_{\alpha}\right)=S_{M S_{\alpha}}
$$

$$
r_{l}+r_{g}=1
$$

Equação da conservação da quantidade de movimento;

$$
\begin{aligned}
\frac{\partial\left(r_{\alpha} \rho_{\alpha} U_{i \alpha}\right)}{\partial t} & +\frac{\partial\left(r_{\alpha} \rho_{\alpha} U_{i \alpha} U_{j \alpha}\right)}{\partial x_{j}} \\
& =-r_{\alpha} \frac{\partial p_{\alpha}}{\partial x_{i}}+\frac{\partial}{\partial x_{i}}\left[r_{\alpha} v_{e f}\left(\frac{\partial U_{j \alpha}}{\partial x_{i}}+\frac{\partial U_{i \alpha}}{\partial x_{j}}\right)\right]+r_{\alpha} \rho_{\alpha} g+F_{\alpha \beta}
\end{aligned}
$$

Modelos de transferência de quantidade de movimento entre a fase líquida e gás.

A transferência de quantidade de movimento entre as fases, $\boldsymbol{F}_{\boldsymbol{\alpha} \boldsymbol{\beta}}$, é medida a partir das forças interfaciais que atuam em cada fase $\alpha$, devido à interação com outra fase $\boldsymbol{\beta}$. Esta força interfacial pode ser expressa por:

$$
F_{l g}=F_{D}+F_{l i f t}+F_{l u b}+F_{V M}+F_{T D}
$$

As forças indicadas acima representam, respectivamente, a força de arraste entre a fase líquida e gás, a força de sustentação, força de lubrificação, força de massa virtual, força de dispersão turbulenta.

A Força de arraste pode ser expressa por:

$$
F_{D}=C_{D} \frac{1}{2} \rho_{L} A_{g}\left(U_{l}-U_{g}\right)^{2}
$$

onde: $\rho_{\mathrm{L}}=$ densidade do líquido; $\left(\mathrm{U}_{\mathrm{L}}-\mathrm{Ug}\right)=$ velocidade relativa; $\mathrm{C}_{\mathrm{D}}=$ coeficiente de arraste; $A_{g}=$ área projetada de uma bolha que pode ser expressa por $\boldsymbol{A}_{\boldsymbol{g}}=\frac{\boldsymbol{\pi}}{\mathbf{4}} \boldsymbol{d}_{\boldsymbol{g}}^{2} ; \mathrm{d}_{\mathrm{g}}=$ diâmetro da bolha. O diâmetro da bolha de gás é: 


$$
d_{g}=0,091\left(\frac{\sigma}{\rho_{l}}\right)^{0,5} u_{g 0}^{0,44}
$$

onde $\sigma=$ tensão superficial e $u_{g 0}=$ a velocidade do gás na saída do injetor.

O fluxo de subida das bolhas no vaso tende a se concentrar numa região próxima à parede. Este efeito pode ser modelado pela força de lubrificação, que tende a expulsar a fase dispersa (argônio) para longe das paredes. O modelo é o Antal cuja força de lubrificação pode ser expressa por:

$$
\boldsymbol{F}_{l u b}=-\boldsymbol{C}_{W} \boldsymbol{r}_{g} \rho_{l}\left|U_{l}-U_{g}\right|^{2} \boldsymbol{n}_{w}
$$

onde: $r_{g}=$ fração volumétrica de gás; $\rho_{L}=$ densidade do líquido; $n_{W}=$ distância normal da parede; $U_{\mathrm{I}}-\mathrm{U}_{\mathrm{g}}=$ diferença de velocidade relativa entre as fase, em um plano próximo a parede.

A força da massa virtual mede o efeito da aceleração do líquido pelo deslocamento das bolhas. E é expressa por:

$$
F_{M V}=\rho_{l} C_{V M} V_{g}\left(d U_{l} d_{t}-d U_{g} / d_{t}\right)
$$

onde $\mathrm{V}_{\mathrm{g}}=$ volume da bolha expresso por $V_{g}=\frac{\pi}{6} d_{g}^{3}$ e $C_{V M}=$ coeficiente de massa virtual igual a 0,5 para bolhas individuais esféricas.

A força de sustentação (lift) é dada em função da velocidade de deslizamento e a curva de velocidade da fase contínua.

$$
F_{L}=\rho_{l} C_{L}\left(U_{l}-U_{g}\right) \times\left(\nabla \times U_{\propto}\right)
$$

Estudos indicam que $C_{L}$ depende do número de Eötvös e pode até assumir valores negativos para bolhas com diâmetros muito grandes.

\subsection{Modelos de Turbulência}

Foram utilizadas dois modelos de turbulência. $O$ modelo $k-\varepsilon$ emprega as equações seguintes para cálculo de viscosidade turbulenta:

Onde:

$$
\begin{aligned}
& \frac{\partial(\rho k)}{\partial t}+\frac{\partial\left(\rho U_{i} k\right)}{\partial x_{i}}=\frac{\partial}{\partial x_{i}}\left[\left(\mu+\frac{\mu_{t}}{\sigma_{k}}\right) \frac{\partial k}{\partial x_{i}}\right]+P_{k}-\rho \varepsilon \\
& \frac{\partial(\rho \varepsilon)}{\partial t}+\frac{\partial\left(\rho U_{i} \varepsilon\right)}{\partial x_{i}}=\frac{\partial}{\partial x_{i}}\left[\left(\mu+\frac{\mu_{t}}{\sigma_{\epsilon}}\right) \frac{\partial \varepsilon}{\partial x_{i}}\right]+\frac{\varepsilon}{k}\left(C_{\varepsilon 1} P_{k}-\rho C_{\varepsilon 2} \epsilon\right)
\end{aligned}
$$

$$
\boldsymbol{P}_{k}=\mu_{t} \frac{\partial U_{j}}{\partial x_{i}}\left(\frac{\partial U_{j}}{\partial x_{i}}+\frac{\partial U_{i}}{\partial x_{j}}\right) \quad \text { e } \quad \mu_{t}=C_{\mu} \rho \frac{k}{\epsilon}
$$

A Tabela 13 apresenta os valores para os coeficientes das equações precedentes.

Tabela 1: Coeficientes das equações dos modelos k- $\varepsilon$ e SST.

\begin{tabular}{ccccc}
$\sigma_{\varepsilon}$ & $\sigma_{\mathrm{\kappa}}$ & $\mathbf{X}_{\varepsilon 1}$ & $\mathrm{X}_{\varepsilon 2}$ & $\mathbf{X}_{\mathrm{u}}$ \\
\hline
\end{tabular}

\subsection{Modelo de Turbulência SST}

O modelo de turbulência SST foi desenvolvido a partir da combinação das vantagens do modelo $k \omega$ nas regiões próximas a uma parede e $k-\varepsilon$ nas regiões mais afastadas da parede. As equações do modelo k- $\omega$ são:

$$
\frac{\partial(\rho k)}{\partial t}+\frac{\partial\left(\rho U_{i} k\right)}{\partial x_{i}}=\frac{\partial}{\partial x_{i}}\left[\left(\mu+\frac{\mu_{t}}{\sigma_{k}^{*}}\right) \frac{\partial k}{\partial x_{i}}\right]+P_{k}-\rho \beta^{\prime} k \omega
$$




$$
\frac{\partial(\rho \omega)}{\partial t}+\frac{\partial\left(\rho U_{i} \omega\right)}{\partial x_{i}}=\frac{\partial}{\partial x_{i}}\left[\left(\mu+\frac{\mu_{t}}{\sigma_{\omega}}\right) \frac{\partial \omega}{\partial x_{i}}\right]+\frac{\omega}{k}\left(\gamma P_{k}-\rho \beta \omega\right)
$$

sendo: $\quad \boldsymbol{P}_{k}=\mu_{t} \frac{\partial U_{j}}{\partial x_{i}}\left(\frac{\partial U_{j}}{\partial x_{i}}+\frac{\partial U_{i}}{\partial x_{j}}\right) \quad$ e $\quad \mu_{t}=\rho \frac{k}{\omega}$

Os valores dos coeficientes das equações anteriores estão listados na Tabela 24.

Tabela 2: Coeficientes das equações do modelo SST.

\begin{tabular}{rrrrr}
\hline$\beta^{\prime}$ & $\sigma^{*_{\mathrm{K}}}$ & $\sigma_{\omega}$ & $\gamma$ & $\beta$ \\
1,3 & 2 & 2 & 0,555 & 0,075 \\
\hline \multicolumn{6}{c}{$\boldsymbol{v}_{\boldsymbol{t}}=\frac{\boldsymbol{a}_{\mathbf{1}} \boldsymbol{K}}{\max \left(\boldsymbol{a}_{\mathbf{1}} \boldsymbol{\omega}, \boldsymbol{s} \boldsymbol{F}_{\mathbf{2}}\right)}$} \\
\end{tabular}

onde: $v_{\tau}=\mu_{\tau} / \rho ; F_{2}=$ função da combinação dos dois modelos; $a_{1}=5 / 9 ; S=$ medida da taxa de cisalhamento.

\section{3- RESULTADOS}

As condições de simulação para estudo do efeito da vazão de sobre a circulação estão elencadas na Tabela 5.

Tabela 5: Casos estudados.

\begin{tabular}{ccl}
\hline Casos & Vazão de Argônio & Modelo de Turbulência \\
\hline 1 & $70 \mathrm{~N} \mathrm{~m}^{3} \mathrm{~h}^{-1}$ & $\mathrm{k}-\varepsilon$ \\
\hline 2 & $90 \mathrm{~N} \mathrm{~m}^{3} \mathrm{~h}^{-1}$ & $\mathrm{k}-\varepsilon$ \\
3 & & SST \\
\hline 4 & $110 \mathrm{~N} \mathrm{~m}^{3} \mathrm{~h}^{-1}$ & $\mathrm{k}-\varepsilon$ \\
5 & & SST \\
\hline 6 & $140 \mathrm{~N} \mathrm{~m}^{3} \mathrm{~h}^{-1}$ & $\mathrm{k}-\varepsilon$ \\
7 & & SST \\
\hline
\end{tabular}

A Figura 1 mostra quatro regiões em corte no reator $\mathrm{RH}$. Os cortes $A-A$ e $B-B$ são horizontais. A-A localiza-se a 0,2 $\mathrm{m}$ abaixo da superfície do aço na câmara de vácuo evidenciando a zona turbulenta próxima à interface de aço líquido/vácuo. Já o corte B-B localizado a 1,0 m do fundo da panela possibilita a visualização dos vórtices que são gerados mais ao fundo da panela, devido à colisão do aço com a panela. Os cortes verticais $C-C$ e $D-D$ representam os planos de simetria das pernas de subida e descida, respectivamente. Vórtices são formados na perna de subida, próximos aos bicos injetores de argônio. Na perna de descida, (Corte D-D), vórtices podem ser formados em decorrência de colisão do aço no fundo da panela. 


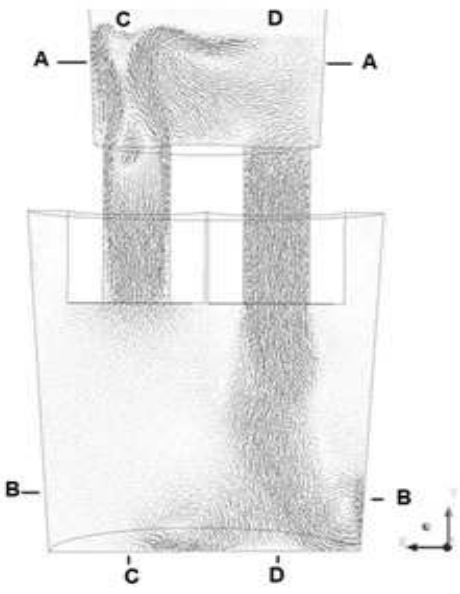

(a)

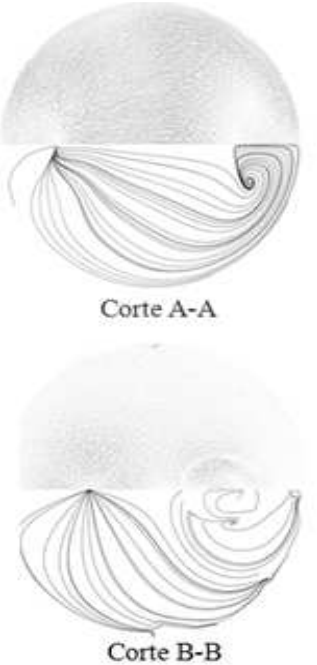

(b)
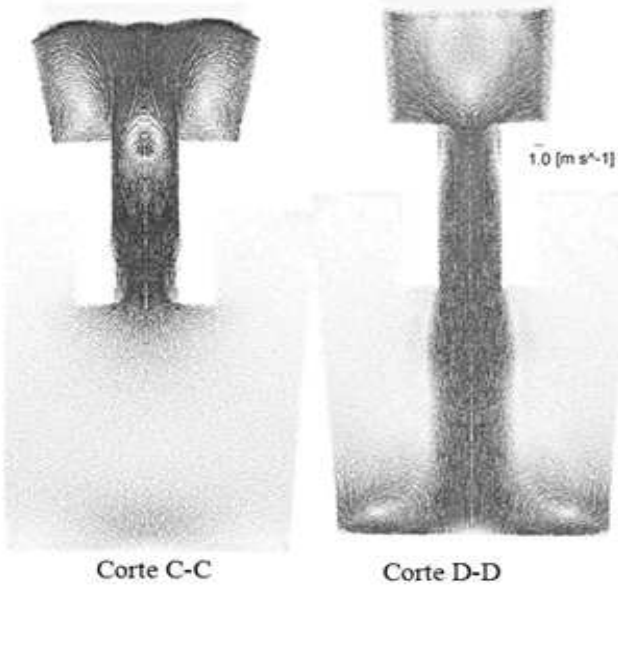

(c)

Figura 1 - (a) Perfil de velocidade no plano de simetria; (b) Perfil de velocidade nos cortes A-A e B-B; (c) Perfil de velocidade nos cortes C-C e D-D (com base no modelo $k-\varepsilon$ ).

Com o aumento da vazão de argônio as plumas gasosas geradas pelos bicos injetores tendem a coalescerem, estimulando a formação de um anel ou coroa de argônio junto à parede interna da perna de subida, onde as velocidades são maiores, Figura 2. O aumento da vazão de argônio incorre em maior profundidade de penetração do jato gasoso em cada um dos bicos injetores; distanciando a coroa gasosa da parede interna da perna de subida. Injeção excessiva de gás pode levar a uma situação na qual a maior parte da área de seção de fluxo seja ocupada por gás, reduzindo a capacidade de bombeamento; entretanto, esta situação não foi detectada nestas simulações.

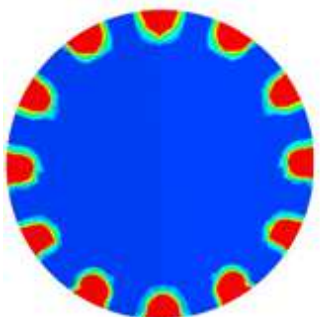

(a)

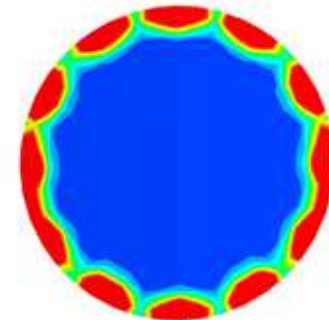

(b)

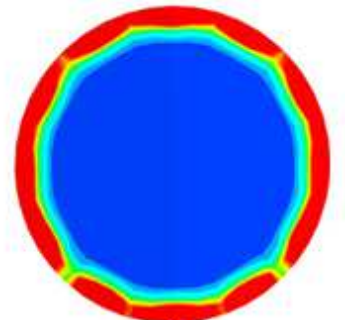

(c)

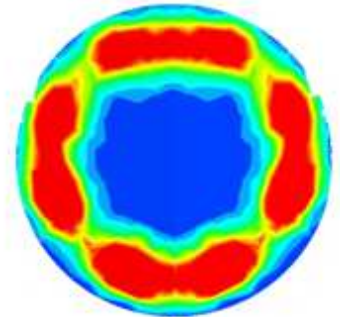

(d)

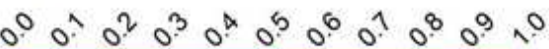

argonio. Volume Fraction

Figura2- Efeito da vazão de argônio sobre a distribuição espacial de gás na perna de subida do reator $\mathrm{RH}$ (com base no modelo $k-\varepsilon$ )

A Figura 3 evidencia que a taxa de dissipação de energia é maior na perna de subida e no fundo da panela, zona de impingimento do jato de aço que escoa pela perna de descida. Nestas regiões, as condições de turbulência favorecem a cinética das reações, além do coalescimento de inclusões. Lacosqui [5] menciona que em um reator com uma panela com capacidade para 320 toneladas de aço e taxa de circulação de $180 \mathrm{ton} / \mathrm{min}$, a taxa de dissipação de energia é da ordem de 0,01 $\mathrm{m}^{2} / \mathrm{s}^{3}$. Para este reator a taxa de dissipação de energia pode ser expressa como:

$$
\varepsilon\left(\mathrm{m}^{2} / \mathrm{s}^{3}\right)=0,008714+0,00969 \frac{\mathrm{Q}}{100} \quad 0,0162 \mathrm{D}_{\text {subida }} \quad 0,0066 \mathrm{D}_{\text {descida }}
$$


onde $\mathrm{Q}=$ vazão de aço, ton $/ \mathrm{min} ; \mathrm{D}_{\text {subida }}=$ área da perna de subida, $\mathrm{m}$ e $\mathrm{D}_{\text {descida }}=$ diâmetro da perna de descida, $m$. Esta expressão ressalta a relação entre taxa de circulação e taxa de dissipação de energia cinética. Burty et al. [6] reportam valores de taxa de dissipação de $0,017 \mathrm{~m}^{2} / \mathrm{s}^{3}$ para taxa de recirculação de $195 \mathrm{ton} / \mathrm{min}$; e taxa de dissipação de $\varepsilon=0,022 \mathrm{~m}^{2} / / \mathrm{s}^{3}$ para taxa de recirculação $=327 \mathrm{ton} / \mathrm{min}$.
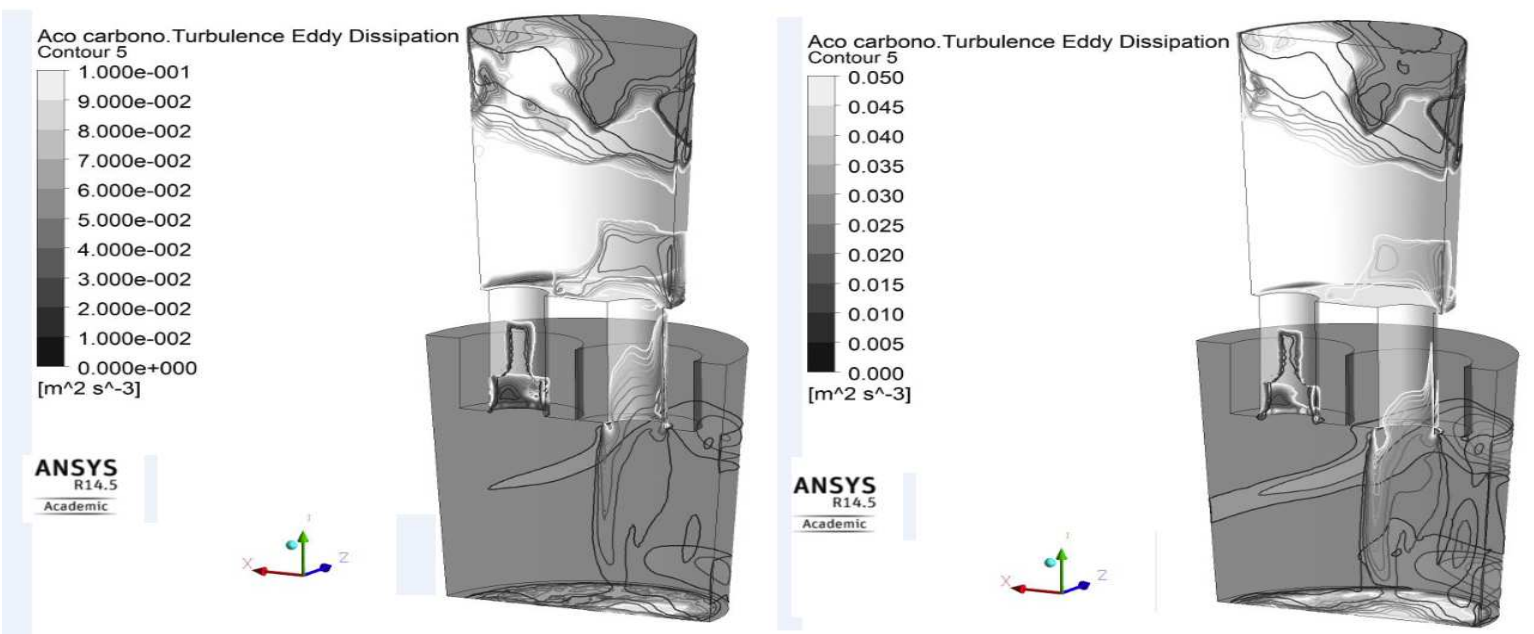

Figura 3 -Taxa de dissipação de energia turbulenta na câmara de vácuo, pernas e panela do reator $\mathrm{RH}$ (com base no modelo $k-\varepsilon$ )

O aumento da vazão de argônio incorre em aumento da taxa de recirculação do aço, Figura 4. No caso de aços ultrabaixo carbono, nos quais as concentrações de hidrogênio, nitrogênio são também muito baixas, a vazão de argônio é $140 \mathrm{Nm}^{3} \mathrm{~h}^{-1}$, o que corresponde a uma taxa de recirculação do aço de: $120 \mathrm{ton} / \mathrm{min}$. (de acordo com Kuwabara [2]), 78ton/min (de acordo com Seshadri [4]) e de 100ton/min (de acordo com este trabalho). Pode-se considerar que o modelo fornece estimativas condizentes com o esperado, desde que se nota grande discrepância entre os valores previstos por fórmulas disponíveis na literatura.

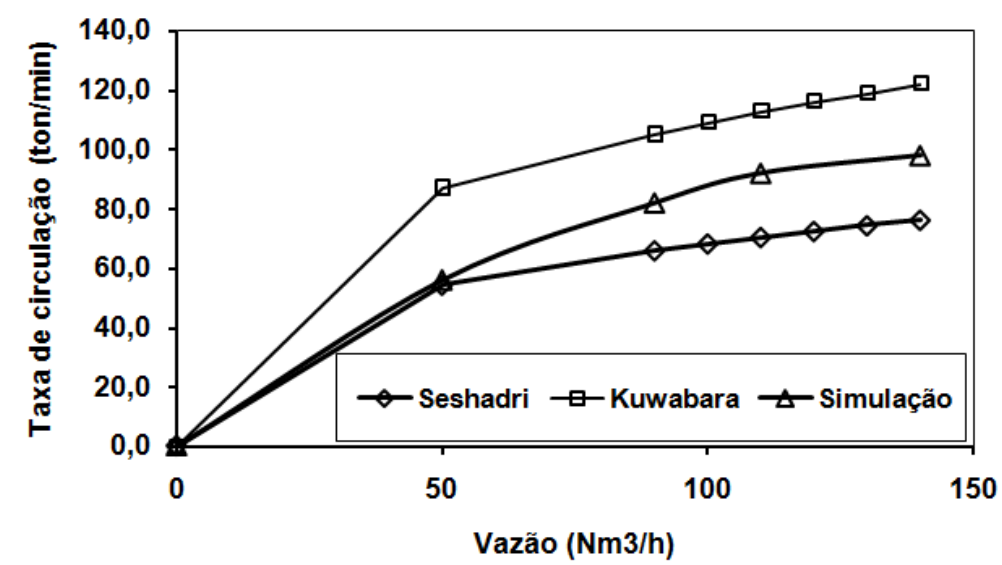

Figura 4 - Variação da taxa de circulação com a vazão de argônio na perna de subida (com base no modelo $k-\varepsilon$ )

\section{4 - DISCUSSÃO}

A taxa de recirculação do aço e a taxa de dissipação de energia são parâmetros que afetam a cinética das reações na interface escória/aço e aço/vácuo. As dimensões, número e formatos das pernas, a vazão de argônio e a pressão no interior da 
câmara de vácuo afetam o campo de velocidades do aço durante o tratamento no $\mathrm{RH}$. Esta análise de fluxo no interior do $\mathrm{RH}$ foi desenvolvida com o objetivo de determinar as condições ótimas para dessulfuração do aço, com adições na câmara de vácuo. Uma série de corridas foi programada para um reator industrial com as características geométricas e operacionais utilizada nesta modelagem matemática. A escória sintética adicionada, via calha na câmara de vácuo, tinha como composição \%CaO = 7máx; \% $\mathrm{Al}_{2} \mathrm{O}_{3}=4$ máx; \%CaF $2=42$ a 48; $\% \mathrm{Cr}_{2} \mathrm{O}_{3}=4$ a 8; $\% \mathrm{SiO}_{2}=10,0$ máx; $\% \mathrm{MgO}=18$ a 24; $\% \mathrm{Fe}_{2} \mathrm{O}_{3}=1,5$ máx; $\% O u t r o s=4,0$ máx; a massa específica aparente desta escória se situa na faixa 1,4 1,6 g/ $\mathrm{cm}^{3}$. A cal empregada tinha como composição desejada \%CaO>93,0; \%P<0,20; \%MgO<2,0; \%S<0,01; \% $\mathrm{SiO}_{2}<2,5$; o diâmetro médio das partículas de cal estava na faixa de 10 20mm. Cal e escória foram adicionados na razão 1:1.

Cálculos preliminares foram realizados para analisar as condições de arraste dos reagentes para a panela e a dessulfuração esperada. Como primeira estimativa das condições de arraste pode ser estabelecido que este será efetivo se a velocidade média do aço na perna de descida for superior à hipotética velocidade de ascensão da partícula de cal no mesmo:

$$
V_{\text {aço }}=\frac{4 Q}{\rho_{\text {aço }} \pi d^{2}} \frac{1}{60} \gg V_{\text {ascensão }}=\sqrt{\frac{8}{3} \frac{R_{p} g \Delta \rho}{\rho_{\text {aço }} f}}
$$

onde $\mathrm{Q}$ é a taxa de circulação (ton/min), d é o diâmetro interno do perna $(\mathrm{m}), \rho_{\text {aço }}$ é a densidade do aço $\left(\mathrm{kg} \cdot \mathrm{m}^{-3}\right), \mathrm{g}$ é a aceleração da gravidade, $\Delta \rho$ é a diferença de densidade entre aço e cal $\left(\mathrm{kg} \cdot \mathrm{m}^{-3}\right), R_{p}$ é o raio da partícula de cal $(\mathrm{m}), f$ é o fator de fricção para fluxo turbulento $\sim 0,44$. Considerando vazão de gás $(G)$ da ordem de $1,833 \mathrm{Nm}^{3} / \mathrm{min}$, perna de diâmetro $d$ igual a $0,65 \mathrm{~m}$, pressão no bico de injeção $\left(\mathrm{P}_{1}\right)$ igual 1bar, pressão na câmara de vácuo $\left(\mathrm{P}_{2}\right)$ igual a 50mbar, a formula de Kuwabara permite determinar a taxa de circulação como

$$
Q(\text { ton } / \mathrm{min})=114 G^{1 / 3} d^{4 / 3}\left(\ln \frac{P_{1}}{P_{2}}\right)^{1 / 3} \sim 113
$$

Com este valor de taxa de circulação e assumindo $\rho_{a c ̧ o}=7000, \Delta \rho=4000$, se encontra um valor crítico de raio da partícula (ou escória) abaixo do qual a mesma é arrastada para a panela:

$$
R=0,02 m
$$

As cargas médias de cal e escória sintética foram da ordem de $1250 \mathrm{~kg}$ cada, para dessulfurar cerca de 240 toneladas de aço. Em função da forte interação provocada pela agitação na câmara de vácuo, seria razoável supor que a escória resultante (líquida) é atomizada e transferida como gotas (menores que $0,02 \mathrm{~m}$ ) até a panela. Após desoxidação profunda os valores de coeficiente de partição de enxofre entre escória e metal são altos; aliado ao fato de que o processo se desenrola em temperaturas altas, se pode assumir que o controle do processo seja por transporte de massa na camada limite do lado do metal, entre metal e gota de escória. Para estimar o coeficiente de transporte de enxofre na camada limite ao redor de uma esfera, Chiang et al. [7], entre muitos ouros, empregaram a correlação:

$$
K(m / s)=\frac{D_{S}}{d}\left[2+1,3\left(\frac{\epsilon^{\frac{1}{3}} d^{\frac{4}{3}} \rho_{a c ̧ o}}{\eta_{a c ̧ o}}\right)^{\frac{1}{2}}\left(\frac{\eta_{a c ̧ o}}{D_{S} \rho_{a c ̧ o}}\right)^{\frac{1}{3}}\right]
$$

onde $D_{S}$ é a difusividade do enxofre no aço, $5 \times 10^{-8} \mathrm{~m}^{2} / \mathrm{s}, d$ é o diâmetro médio da gota arrastada até a panela $(\mathrm{m}), \rho_{\text {aço }}$ é a densidade do aço, $7000 \mathrm{~kg} / \mathrm{m}^{3}$, $\eta_{\text {aço }}$ é a 
viscosidade do aço, 0,006Pa.s, e $\epsilon$ representa a taxa de dissipação de energia cinética de turbulência $\mathrm{m}^{2} / \mathrm{s}^{-3}$.

Através da modelagem matemática, uma relação entre vazão de gás, taxa de circulação e taxa (média sobre o volume da panela) de dissipação de energia cinética de turbulência, ver Tabela 5 . Como se nota a partir da Figura 3 existe uma ampla variação de valores desta grandeza, os maiores se concentrando nas pernas e câmara de vácuo; os valores na panela são comparativamente muito menores.

Tabela 5: Valores de taxa de dissipação de energia cinética de turbulência obtidos.

\begin{tabular}{|c|c|c|}
\hline Vazão de gás $\mathrm{Nm}^{3} / \mathrm{min}$ & Circulação ton/min & Dissipação de energia na panela $\mathrm{m}^{2} / \mathrm{s}^{3}$ \\
\hline 110 & 94 & $1,3 \times 10^{-4}-1,1 \times 10^{-3}$ \\
\hline 140 & 98 & $2,5 \times 10^{-4}-1,4 \times 10^{-3}$ \\
\hline 180 & 120 & $5,8 \times 10^{-4}-2,1 \times 10^{-3}$ \\
\hline
\end{tabular}

Para gotas com diâmetro estimado como $d=8 \times 10^{-3} \mathrm{~m}$ (o que corresponde a 1/5 do valor crítico) e taxa de dissipação $\epsilon=0,02$, se encontra o valor de coeficiente de transporte de massa que deve ser aplicado à superfície total das gotas de escória imersas no aço,

$$
K(\mathrm{~m} / \mathrm{s})=4,85 \times 10^{4} \quad \text { e } \quad A=\frac{6 \mathrm{M}}{\rho_{\text {escória }} \mathrm{d}}=625 \mathrm{~m}^{2}
$$

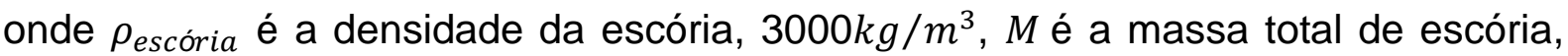
$2500 \mathrm{~kg}$, e d o diâmetro presumido de cada gota, $8 \times 10^{-3} \mathrm{~m}$. A Tabela 6 elenca dados da operação de de-S do aço no reator $\mathrm{RH}$.

Tabela 6: Dados do tratamento de dessulfuração do aço líquido no $\mathrm{RH}$

\begin{tabular}{|c|c|c|c|c|c|c|c|c|c|}
\hline & $\begin{array}{c}\text { Temperatur } \\
\text { a (panela) }\end{array}$ & Al & $\begin{array}{c}\text { Escória } \\
\text { LD }\end{array}$ & Cal & $\begin{array}{c}\text { Escória } \\
\text { Sintética }\end{array}$ & $\begin{array}{c}\text { [\%S }]_{0} \\
\text { Corrida }\end{array}$ & $\begin{array}{l}\text { Eficiência } \\
\text { de De-S }\end{array}$ & \multicolumn{2}{|c|}{ Parâmetros RH } \\
\hline 1 & 1608 & 0,037 & 2,5 & 1300 & 1300 & 190,8 & 26,62474 & 50 & 110 \\
\hline 2 & 1596 & 0,052 & 1,9 & 1250 & 1250 & 135,7 & 41,04643 & 50 & 110 \\
\hline 3 & 1591 & 0,054 & 1,4 & 1250 & 1250 & 89,4 & 44,07159 & 50 & 110 \\
\hline 4 & 1647 & 0,043 & 1,1 & 1250 & 1231 & 82,1 & 26,91839 & 50 & 110 \\
\hline 5 & 1586 & 0,04 & 2,2 & 1229 & 1250 & 115 & 30,43478 & 50 & 110 \\
\hline 6 & 1621 & 0,04 & 2 & 1281 & 1250 & 108 & 25,92593 & 50 & 110 \\
\hline 7 & 1584 & 0,04 & 1,9 & 1281 & 1250 & 115 & 39,13043 & 50 & 110 \\
\hline
\end{tabular}

O tempo de circulação é da ordem de 6 minutos e presume-se que parte das gotas flota e é absorvida pela escória de cobertura da panela. Por este motivo se assume um tempo médio de contato de 1 minuto. Neste intervalo a quantidade de enxofre que é transferida do metal até a escória é da ordem de

$$
\Delta S(k g)=A \cdot K \cdot \frac{\% S}{100} \rho_{a c ̧ o} \Delta t
$$

onde \%S representa a concentração inicial, em peso, de enxofre e $\Delta t \mathrm{~s}$ o tempo de contato. Então, para \%S da ordem de 0,01 o grau de de-S corresponde a um transporte de cerca de $12,7 \mathrm{~kg}$ de enxofre até as gotas de escória. Comparativamente ao teor inicial de enxofre a taxa de de-S seria da ordem de $50 \%$. Estes cálculos são aproximados, pois não consideram a participação da escória de cobertura na panela, a distribuição de tamanhos e de tempos de residência das gotas na panela e a eventual reação na câmara de vácuo, onde as condições de 
agitação são mais intensas (e os tempos de residência mais curtos). Os valores médios de de-S obtidos nestes experimentos foram próximos de $35 \%$. O que pode ser tomado como comprovação da análise efetuada.

\section{5 - CONCLUSÕES}

O aumento da vazão de argônio incorreu em aumento na taxa de circulação do aço. A modelagem matemática produz resultados consistentes com os esperados nas operações industriais. Os resultados de de-S encontrados na prática industrial se aproximam dos valores antecipados, por análise baseada na computação numérica do fluxo

\section{Agradecimentos}

\section{FAPEMIG e CNPq.}

\section{LISTA DE VARIÁVEIS}

$\mathrm{S}_{\mathrm{MS} \alpha}$ : termo fonte de massa específica;

$\mathrm{S}_{\mathrm{Ma}}$ : termo fonte da quantidade de movimento

$F_{\alpha \beta}$ : força interfacial que atua na fase $\alpha$ devido à presença da fase $\beta$. O termo $\left(\Gamma_{\alpha \beta}^{+} U_{\beta}-\Gamma_{\beta \alpha}^{+} \boldsymbol{U}_{\alpha}\right)$ taxa de transporte de quantidade de movimento devida à transferência de massa entre as fases.

\section{REFERÊNCIAS}

1 ZHANG,J; LIU,L; ZHAO, X; LEI,S \& DONG, Q - Mathematical Model for Decarburization Process in RH Refining Process ISIJ International, Vol. 54 (2014), No. 7, pp. 1560-1569

2 KUWABARA, T; UMEZAWA, K; MORI, K \& WATANABE, $\mathrm{H}$ - Investigation of decarburization behavior in $\mathrm{RH}$-Reactor an its operation improvement. Transactions ISIJ, 28, 1981, pp. 305-314

3 SILVA, C.A; SILVA. I.A ; MARTINS, E.M.C; SESHADRI,V; PERIM, C.A \& VARGAS FILHO, G.A - Fluid flow and mixing characteristics in RH degasser of Companhia Siderúrgica de Tubarão, and influence of bottom gas injection and nozzle blockage through physical modelling study. Ironmaking Steelmaking, 31, 2004, pp. 37-42

4 SESHADRI, V \& COSTA, S.L.S., Cold Model of RH Degassing, Transactions of ISIJ, Vol.26, p.133-138,1986.

5 LACOSQUI, P.S.B - Avaliação da Limpidez do Aço Líquido Através da Modelagem Matemática do Desgaseificador - RH da Companhia Siderúrgica de Tubarão - CST. Dissertação de Mestrado, Redemat, UFOP, 2007

6 BURTY, M; BOHER, M; de SANTIS, M; MATKZEIT, M \& GUDENAU, H.W Improvement of inclusions flotation during $\mathrm{RH}$ treatment. Technical Steel Reearch, Steelmaking. European Commission, 2006. 116 gages

7 CHIANG, L.K; IRONS, G.A. LU, W.K \& CAMERON, I.A Kinetic studies of calcium carbide hot metal desulfurization by powder injection; Transactions ISS, 1990, p. 1-18 Manual do ANSYS-CFX 15 